\title{
Recurrence of measles in central Italy: the experience of a hospital in Rome
}

\author{
Andrea Petrucca, Antonella Alari, Styliani Papadopoulou, Cristina Petrucci, Iolanda Santino \\ Department of Molecular and Clinical Medicine, School of Medicine and Psychology, Sapienza University of Rome; \\ Microbiology Unit, Sant'Andrea University Hospital, Rome, Italy
}

\section{Summary}

Measles continue to be a major public health issue worldwide with high morbidity and mortality rates. The disease is still endemic in Europe and during 2017 a vast outbreak was described in Italy, Romania and Hungary, which led to thousands of new cases and several deaths. In Italy, 3931 confirmed cases of measles were reported to the Italian national surveillance system from many Italian administrative regions; Lazio, in central Italy, exhibited the highest number of infected patients 1322 (33.63\%) and as well as the highest incidence.

In this study, we describe the results of a retrospective analysis, carried out during 2016 and 2017, concerning the measles antibody prevalence in patients and healthcare workers attending the Sant'Andrea Hospital of Rome (Lazio).

A total of 94 patients (median 30 years of age) were screened in 2016, and 316 (median 40 years of age) during 2017, with an increase of $236 \%$ compared to previous year. During 2017, 41 confirmed cases of measles were reported while none in 2016

Correspondence: Iolanda Santino, Department of Molecular and Clinical Medicine, School of Medicine and Psychology, Sapienza University of Rome, Microbiology Unit, Sant'Andrea University Hospital, Via di Grottarossa 1035, 00189 Rome, Italy.

Tel.: +39.0633775488 - Fax: +39.0633776694.

E-mail: iolanda.santino@uniroma1.it

Key words: measles, serological diagnosis, surveillance, patients, healthcare workers.

Contributions: AP, AA, SP, CP, data collecting and references search; $\mathrm{AP}$, data analysis and manuscript writing; IS, manuscript writing and reviewing.

Conflict of interests: the authors declare no potential conflict of interests.

Funding: none.

Received for publication: 14 December 2018.

Revision received: 8 February 2019.

Accepted for publication: 14 February 2019.

CC Copyright A. Petrucca et al., 2019

Licensee PAGEPress, Italy

Microbiologia Medica 2019; 34:7999

doi:10.4081/mm.2019.7999

This article is distributed under the terms of the Creative Commons Attribution Noncommercial License (by-nc 4.0) which permits any noncommercial use, distribution, and reproduction in any medium, provided the original author(s) and source are credited.
$(\mathrm{P}<0.007)$, and we found a suboptimal immunization coverage in our cohort of patients. Furthermore, measles surveillance of Sant'Andrea healthcare workers during the study period involved 208 personnel units (median $>47$ years of age) and only one confirmed measles infection was recorded in 2017.

These results suggest that there is still an unvaccinated portion of the adult population, who sustain the endemic circulation of measles in Italy. In addition to reach herd immunization on children of 2 years old, catch-up vaccination campaign targeting adult population in Italy and other European countries needs to be implemented to prevent future measles outbreak.

\section{Introduction}

Measles virus (MV) is a member of the genus Morbillivirus (Paramyxoviridae family), which causes measles in humans, an acute viral disease characterized by high fever, conjunctivitis, coryza, cough and a maculopapular rash often associated with severe complications like pneumonia, encephalitis and in some cases patients' death $(9,14)$.

Worldwide, measles morbidity and mortality have been reduced since the implementation of enhanced vaccination strategies $(2,6)$, and in several countries the interruption of indigenous transmission of measles disease has been reported (17). Unfortunately, the measles disease continues to be difficult to eradicate especially in European countries where recurrent outbreaks have been described $(16,17)$. It is valuable to highlight that during 2013, Turkey, Russian Federation, United Kingdom, Italy, Ukraine and Germany (countries with $>1.000$ cases) reported 15.405 measles infection $(81.5 \%)$ out of 18.907 cases ascertained in Europe (3).

In Italy, even though a significant reduction of measles infection has been obtained, since the introduction of the measles vaccine in 1976, the goal of measles eradication fixed by WHO European region in 2010 and 2015, has still not been met $(4,15-$ 17). The main reason for these negative results is related to the vaccination rates that never reached the coverage of $95 \%$ in children below 2 years of age. In this population, during 2000s the vaccine coverage ranged from $75 \%$ to $88 \%$, and only in 2003 and 2017 it reached the highest level of 90.8 and $91.68 \%$, respectively (8). Likewise, in 2017 vaccination coverage of 4-, 5-6 and 8-yearold children ranged from 85 to $90 \%$ while young adult of 16 to 18 years were just above $80 \%$ (8). However, each regions of Italy has shown extremely variable vaccination coverage with the lowest level affecting regions of central and southern Italy (8).

During 2017, a large outbreak of measles was described in Italy, Romania and Hungary, which led to thousands of new cases and several deaths (12). Regarding Italy, 3931 confirmed cases had been reported to the Italian surveillance system (ISS), with two cases of encephalitis and three deaths (7). Most of the Italian 
reports were made in Piedmont, Lombardy, Tuscany, Lazio and Abruzzo regions. In particular, Lazio is the central area of Italy that showed both the highest number of confirmed cases 1322 out of the 3931 reported $(33.63 \%)$, has well as the highest incidence 28.8 $\times 100.000$ habitants $(7)$.

The aim of this study is to report from January 2016 to December 2017 the prevalence of measles antibodies in patients and healthcare workers attending at Sant'Andrea Hospital of Rome.

\section{Materials and Methods}

\section{Study population, serological test and statistical analysis}

A retrospective study focused on studying the measles seroreactivity was conducted, between January 2016 and December 2017, at the Microbiology Unit of Sant'Andrea University Hospital, Rome, Italy. During 2016, an overall number of 94 serum samples were analysed, while for 2017 , the number was 316 . Out of these numbers, 47 and 161 were samples obtained from Sant'Andrea healthcare workers screened for measles immunity in 2016 and 2017, respectively.

Sera were tested by a chemiluminescence immunoassay (Liaison XL analyser, Diasorin, Italy), for detection of measles virus IgM and IgG antibodies, following manufacturer's instructions. Results were subjected to $\chi^{2}$ analysis (significance at $\mathrm{P}<0.05$ ) to compare the frequency distribution. All patients with measles IgM antibody were considered laboratory confirmed cases and the results were reported to the Sant'Andrea Infectious Disease Unit. An association between a laboratory measles IgM finding and a patient's clinical condition (i.e. fever, cough, maculopapular rush, coryza and conjunctivitis), was related to a confirmed case of infection.

\section{Results}

An overall number of 94 patients were screened in 2016, out of whom 61 were female $(65 \%)$ and 33 male $(35 \%)$. The median age was 30 years, ranging from 2 to 63 years of age (Table 1). The great majority of the 2016 patient cohort were outpatients coming from the Phlebotomy Department of Sant'Andrea Hospital. Serological measles IgG reactivity showed that $87 \%$ (82 patients) were positive for $\mathrm{IgG}$, thus showing immunological protection while $13 \%$ (12 patients) tested negative.

In particular, 7 out of the 12 measles $\operatorname{IgG}$ negative patients enrolled in 2016 were born before introduction in Italy of measles vaccine in 1976 (Table 2). Regarding measles IgM reactivity, no measles infection was ascertained in Sant'Andrea Hospital during 2016 (Table 2).

During 2017, we tested serum samples of 316 patients with an increase of $236 \%$ compared to previous year. The population characteristics were similar to that of 2016 with 217 female (68\%) and 99 male (32\%). The median age was 40 years, ranging from 2 to 82 years (Table 1). Seroprevalence for measles IgG among our 2017 patient cohort was 78\% (248) with immunological protection and $22 \%$ (68) without. Among measles IgG negative patients, in 2017 we observed that 39 of them were born before 1976 (Table 2). An overall number of 189 out of 316 patients were screened for measles IgM, and $21 \%$ (40) of whom were positive and considering their clinical status were, classified as confirmed cases (Table 2). It was interesting to note that during 2017, physicians for some unknown reasons did not request a full measles antibodies screening for 129 patients (Table 2). These patients have been screened only for measles IgG antibodies, therefore other IgM positive patient may have been misdiagnosed.

As the number of confirmed measles infection, considerably increase during 2017, Sant'Andrea Hospital Management intensified its measles surveillance programme among hospital employees. An overall of 208 healthcare workers, 47 in 2016 and 161 in 2017 (median age of 47 and 49 years, respectively) were screened for measles $\operatorname{IgG}$ antibodies (Tables 1 and 2). This amounted to a $243 \%$ increase in screening from 2016 to 2017. Analysis of the healthcare employees' measles immunity showed that $74 \%$ and $71 \%$ of them were positive in 2016 and 2017 respectively, while only one $\operatorname{IgM}$ reactivity was detected in 2017 (Table 2).

Table 1. Characteristics of study population.

\begin{tabular}{lcccc} 
& & Patients & & Healthcare workers \\
& 2016 & 2017 & 2016 & 2017 \\
Serum sample & 94 & 316 & 47 & 161 \\
Male/female & $33 / 61$ & $99 / 217$ & $12 / 35$ & $49 / 112$ \\
\hline Median age & 30 (range: $2-63$ years) & 40 (range: $2-82$ years) & 47 (range: $34-62)$ & 49 (range: $32-64)$ \\
\hline
\end{tabular}

Table 2. Seroprevalence of IgG and IgM of patients and healthcare workers analysed in 2016 and 2017.

\begin{tabular}{|c|c|c|c|c|c|c|c|c|c|}
\hline & \multicolumn{4}{|c|}{ Patients } & \multicolumn{4}{|c|}{ Healthcare workers } \\
\hline & & \multicolumn{2}{|c|}{$\operatorname{IgG}$} & \multicolumn{2}{|c|}{ IgM } & \multicolumn{2}{|c|}{$\operatorname{Ig} G$} & \multicolumn{2}{|c|}{$\operatorname{Ig} M$} \\
\hline & & Positive (\%) & Negative (\%) & Positive (\%) & Negative (\%) & Positive (\%) & Negative (\%) & Positive (\%) & Negative (\%) \\
\hline \multirow[t]{3}{*}{2016} & Total & $82(87)$ & $12(13)$ & - & $94(100)$ & $35(74)$ & $12(26)$ & - & $47(100)$ \\
\hline & Born before 1976 & $35(36)$ & $7(7)$ & - & $42(44)$ & $34(72)$ & $10(22)$ & - & $44(94)$ \\
\hline & Born after 1976 & $47(51)$ & $5(5)$ & - & $53(56)$ & $1(2)$ & $2(4)$ & - & $3(6)$ \\
\hline \multirow[t]{3}{*}{2017} & Total & $248(78)^{a}$ & $68(22)$ & $40(21)^{b}$ & $149(79)$ & $114(71)$ & $47(29)$ & $1(0.6)$ & $160(99.4)$ \\
\hline & Born before 1976 & $117(37)$ & $39(13)$ & $28(15)$ & $82(44)$ & $102(63)$ & $42(26)$ & $1(0.6)$ & $143(89)$ \\
\hline & Born after 1976 & $131(41)$ & $29(9)$ & $12(6)$ & $67(35)$ & $12(8)$ & $5(3)$ & - & $15(9.4)$ \\
\hline
\end{tabular}

aStatistical analysis comparing measles IgG seroprevalence of 2017 vs. 2016 showed a $\mathrm{P}<0.01$; b ${ }^{b}$ Statistical analysis comparing measles IgM seroprevalence of 2017 vs. 2016 showed a $\mathrm{P}<0.007$. 


\section{Characteristics of $\mathbf{4 1}$ patients with laboratory confirmed measles infection}

Patients with laboratory confirmed measles infection were 19 female (46\%) and 22 male (54\%). By studying the age distribution of IgM positive patients, we observed that the highest percentage of them $(67 \%)$ were in a population group of 26-50 years followed by individuals of $13-26$ years $(18 \%)$ and $0-12$ years $(10 \%)$. The remaining $5 \%$ were in individuals over the age of 50 (Figure 1). Of the 41 patients with measles infection, $21 \%$ were outpatients who went to the Phlebotomy Department of Sant'Andrea Hospital of Rome while the remaining 79\% were admitted to Emergency Department. The latter group exhibited a plethora of signs and symptoms spanning from a cutaneous rash to fever and diarrhoea and, in some circumstances, respiratory insufficiency. Once the measles infection was laboratory and clinical confirmed, inpatients were transferred to isolation rooms within the Emergency Department of Sant'Andrea Hospital, and then moved to the Infectious Disease Department of other hospital. There was scarce information about the vaccination status of in- and outpatients, but the great majority of them referred do not to remember if they had gotten a measles vaccination. Two cases reported in this study occurred within the same family and involved a 5-year old daughter that had not received the $2^{\text {nd }}$ vaccination dose yet and her susceptible father. Based to the measles surveillance among healthcare workers, in 2017, we found one confirmed infection where the transmission route was not well-understood.

\section{Discussion and Conclusions}

Measles is a highly contagious viral disease that is preventable with vaccination, in the absence of preventive measures, the disease will develop in more than $90 \%$ of susceptible persons when they become exposed to infected patients (13). Measles elimination programs were based on maintaining optimal levels of vaccination coverage and implementation of a national and a local surveillance system. In this contest, laboratory surveillance is an integral part of the global eradication program (11).

In this study, we described the results of a retrospective analysis, carried out during 2016 and 2017, concerning measles antibody prevalence in patients presented to the Sant'Andrea Hospital of Rome (Lazio). Our results, in line with the national ones, have confirmed the epidemic event we observed in 2017. There was a $236 \%$ increase in the number of requests for measles infection

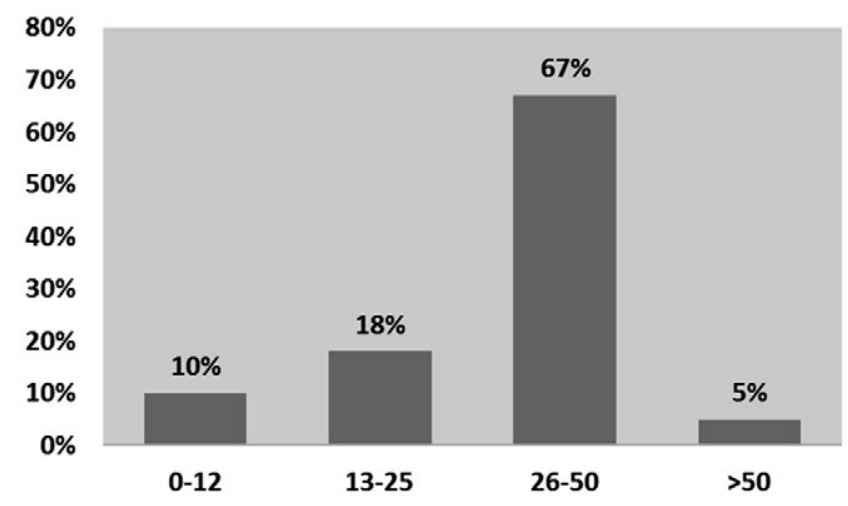

Figure 1. Measles confirmed cases $(n=41)$ distributed by age groups. serodiagnosis, from 94 requests in 2016 to 316 in 2017. With regard to the cases of established infections, in 2016 none of the subjects analysed were infected by measles, while in 2017 there was a statistically significant increase of cases with 40 infected patients $(\mathrm{P}<0.007)$ and 1 healthcare worker.

The $67 \%$ of measles infected patients were in the group of $26-$ 50 years, while only $10 \%$ of cases were observed in children of 0 12 years. This is in accordance with the data reported in 2017 at national and at European level, where most cases (74\% and 45\%, respectively) were described in people aged 15 years and over (3, $8)$. In agreement with this evidence, if we analyse IgG seroprevalence of Sant'Andrea patients and healthcare workers, we find that an increase in age corresponds to a decrease in measles immunization coverage (Table 2). An analysis performed on measles epidemiological data retrieved from EpiCentro web site (http://www.epicentro.iss.it/problemi/morbillo/epidItalia.asp), shows that from 1984 to 1990 only $20 \%$ of children aged 2 years (born from 1982 to 1988 and now $>30$ years old) were vaccinated, a percentage that increased up to $50 \%$ in 1998 . Unfortunately, data for previous years are not available but it is reasonable to expect that the percentages are still lower. This means, that a large percentage of people born up to the 1990s, are not immunized against measles and now represent an immense reservoir susceptible to infection (8 and http://www.epicentro.iss.it/problemi/morbillo/ epidItalia.asp). The data presented in this report seems to confirm this trend, showing that the great majority of patients not immunized against measles were born before 1976 (Table 2). This high number of unimmunized adults against measles, helps explain the prevalence and increase frequency of outbreaks after 1999 (20022003, 2008, 2010-2011, 2013-2014 and 2017), despite the fact that vaccination coverage reached a stable level of $>80 \%$ after 1999 (http://www.epicentro.iss.it/problemi/morbillo/epidItalia.asp).

To date, achieving herd immunity, by extensively immunizing not only children but also adults is the only effective weapon that we can use to break the spread of measles virus.

The measles outbreak of 2017 involved 302 health workers in Italy (5). This result was not unexpected since an analysis of the Italian national measles surveillance data from October 2010 to December 2011 showed that healthcare workers accounted for $11.6 \%$ of the reported cases (4).

During 2016, in our hospital, we undertook a low-level healthcare workers' surveillance involving 47 units of personnel. In concomitance with the measles epidemic peak of 2017, this number was greatly enhanced with 161 workers examined $(+243 \%)$. In our data, we found as immunization coverage (74\%) that was far lower than the data reported by the Italian National Health Institute (ISS), for Italian population (87\%) (http://www.epicentro.iss.it/proble$\mathrm{mi} / \mathrm{morbillo} /$ epidItalia.asp). Median age of examined Sant'Andrea healthcare workers was 47 and 49 years in 2016 and 2017, respectively, meaning that a significative subset of workers were born before 1976 when measles the vaccination was introduced (Table 2 ). In this age group, measles IgG seroprevalence was $72 \%$ and $63 \%$ during 2016 and 2017 respectively, while more than $26 \%$ of examined Sant'Andrea healthcare workers showed no immunization against measles (Table 2). Nosocomial transmission of measles has been frequently described in literature and its one of the most important routes of infection $(1,10)$. During 2017, only one Sant'Andrea hospital worker showed a measles infection, but no transmission was ascertained among other healthcare workers, inpatients or within members of his family. While the lack of measles transmission was an extremely lucky and unlikely occurrence that could have turned differently, it must remind us that the measles immunization is highly needed for all susceptible healthcare workers and appropriate infection control measures need to be 
in place in hospitals to minimize nosocomial spread of infection.

In conclusion, measles is a disease that should not be underestimated, as it can has serious consequences for health. Given that, it can be easily prevented through a complete cycle with a twodose vaccination (the only effective prevention measure) and every effort should be made to increase immunization rates of the unimmunized population. Collectively all the data discussed above suggest that there is still a not-immunised portion of the adult population, who sustain the endemic circulation of measles in Italy. In order to reach the "herd immunity" in children of 2-year-old, and to prevent future measles outbreak catch-up vaccination campaign targeting infant, adolescent and adult population in Italy and other European countries needs to be urgently take place.

\section{References}

1. Botelho-Nevers E, Gautret P, Biellik R, et al. Nosocomial transmission of measles: an updated review. Vaccine 2012;30:3996-4001.

2. Coughlin MM, Beck AS, Bankamp B, et al. Perspective on Global Measles Epidemiology and Control and the Role of Novel Vaccination Strategies. Viruses 2017;19: 9.

3. European Centre for Disease Prevention and Control (ECDC). Monthly measles epidemiological updates. Available from: https://ecdc.europa.eu/en/publications-data/measles-affectsall-age-groups Accessed: November 2018.

4. Filia A, Bella A, Rota MC, et al. Analysis of national measles surveillance data in Italy from October 2010 to December 2011 and priorities for reaching the 2015 measles elimination goal. Euro Surveill 2013;18:20480.

5. Filia A, Bella A, Del Manso M, et al. Ongoing outbreak with well over 4,000 measles cases in Italy from January to end August 2017 - what is making elimination so difficult? Euro Surveill 2017;22:1-5.

6. Greenwood B. The contribution of vaccination to global health: past, present and future. Philos Trans R Soc Lond B Biol Sci. 2014;369:20130433.

7. Istituto Superiore di Sanità, Epicentro. Morbillo \& Rosolia News. Sorveglianza Integrata del Morbillo e della Rosolia.
Rapporto N ${ }^{\circ} 37$ - Gennaio 2018. Available from: www.epicentro.iss.it/problemi/morbillo/bollettino/RM_News_2018_37\%2 0def.pdf Accessed: November 2018.

8. Italian Ministry of Health $(\mathrm{MoH})$. Vaccinazioni dell'età pediatrica e dell'adolescente. Coperture vaccinali. [Childhood and adolescent vaccinations. Vaccine coverage]. Rome: $\mathrm{MoH} ; 2017$. Available from: http:/www.salute.gov.it/portale/documentazione/p6_2_8_3_1.jsp?lingua $=$ italiano\&id $=20$ Accessed: November 2018 .

9. Laksono BM, de Vries RD, McQuaid S, et al. Measles Virus Host Invasion and Pathogenesis. Viruses 2016;8:210.

10. Maltezou HC, Wicker S. Measles in healthcare settings. Am J Infect Control 2013;41:661-3.

11. Orenstein WA, Cairns L, Hinman A, et al. Measles and Rubella Global Strategic Plan 2012-2020 midterm review report: Background and summary. Vaccine 2018;36:35-42.

12. Orosz L, Gáspár G, Rózsa Á, et al. Epidemiological situation of measles in Romania, Italy, and Hungary: On what threats should we focus nowadays? Acta Microbiol Immunol Hung 2018;65:127-34.

13. Rasmussen LD, Fonager J, Knudsen LK, et al. Phylogenetic and epidemiological analysis of measles outbreaks in Denmark, 2013-2014. Euro Surveill 2015;20:30027.

14. Rota PA, Moss WJ, Takeda M, et al. Measles. Nat Rev Dis Primers 2016;2:1-16.

15. Williams GA, Bacci S, Shadwick R, et al. Measles among Migrants in the European Union and the European Economic Area. Scandinavian Journal of Public Health 2016;44:6-13.

16. World Health Organization (WHO) Regional Office for Europe. Sixth Meeting of the European Regional Verification Commission for Measles and Rubella Elimination (RVC), 1517 June 2017, Bucharest, Romania. Copenhagen: WHO Regional Office for Europe 2017. Available from: http://www.euro.who.int/_data/assets/pdf_file/0019/348013/6t h-RVC-final-for-web-posting.pdf?ua=1 Accessed: November 2018.

17. World Health Organization. Measles no longer endemic in $79 \%$ of the WHO European Region. 2017 Available from: http://www.euro.who.int/en/media-centre/sections/pressreleases/2017/measles-no-longer-endemic-in-79-of-the-whoeuropean-region. Accessed: November 2018. 